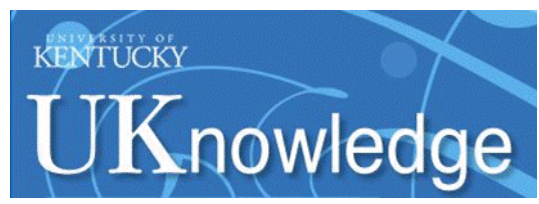

University of Kentucky

UKnowledge

\title{
Atomic Data for Astrophysics. I. Radiative Recombination Rates for H-like, He-like, Li-like, and Na-like Ions over a Broad Range of Temperature
}

D. A. Verner

University of Kentucky

Gary J. Ferland

University of Kentucky, gary@uky.edu

Follow this and additional works at: https://uknowledge.uky.edu/physastron_facpub

Part of the Astrophysics and Astronomy Commons, and the Physics Commons

Right click to open a feedback form in a new tab to let us know how this document benefits you.

\section{Repository Citation}

Verner, D. A. and Ferland, Gary J., "Atomic Data for Astrophysics. I. Radiative Recombination Rates for Hlike, He-like, Li-like, and Na-like lons over a Broad Range of Temperature" (1996). Physics and Astronomy Faculty Publications. 152.

https://uknowledge.uky.edu/physastron_facpub/152

This Article is brought to you for free and open access by the Physics and Astronomy at UKnowledge. It has been accepted for inclusion in Physics and Astronomy Faculty Publications by an authorized administrator of UKnowledge. For more information, please contact UKnowledge@lsv.uky.edu. 
Atomic Data for Astrophysics. I. Radiative Recombination Rates for $\mathrm{H}$-like, Helike, Li-like, and Na-like Ions over a Broad Range of Temperature

Digital Object Identifier (DOI)

http://dx.doi.org/10.1086/192284

Notes/Citation Information

Published in The Astrophysical Journal Supplement Series, v. 103, no. 2, p. 467-473.

(C) 1996. The American Astronomical Society. All rights reserved.

The copyright holder has granted permission for posting the article here.

This article is available at UKnowledge: https://uknowledge.uky.edu/physastron_facpub/152 
The Astrophysical Journal SUPPlement Series, 103:467-473, 1996 April

(@) 1996. The American Astronomical Society. All rights reserved. Printed in U.S.A.

ATOMIC DATA FOR ASTROPHYSICS. I. RADIATIVE RECOMBINATION RATES FOR H-LIKE, He-LIKE, Li-LIKE, AND Na-LIKE IONS OVER A BROAD RANGE OF TEMPERATURE

\author{
D. A. VERNER AND G. J. FERLAND \\ Department of Physics and Astronomy, University of Kentucky, Lexington, KY 40506; verner, gary@pa.uky.edu \\ Received 1995 July 14; accepted 1995 September 21
}

\begin{abstract}
We present new calculations and analytic fits to the rates of radiative recombination toward $\mathrm{H}$-like, $\mathrm{He}$-like, $\mathrm{Li}$ like, and Na-like ions of all elements from $\mathrm{H}$ through $\mathrm{Zn}(Z=30)$. The fits are valid over a wide range of temperature, from $3 \mathrm{~K}$ to $10^{9} \mathrm{~K}$.

Subject headings: atomic data - atomic processes
\end{abstract}

\section{INTRODUCTION}

Astrophysical plasmas are generally dilute and far from thermodynamic equilibrium. As a result their physical state and resulting spectra are determined by the balance set by a host of microphysical processes. Total recombination rate coefficients are essential for the prediction of the ionization balance, among other things. Recombination at low densities proceeds through three different physical processes: radiative recombination, $\mathrm{A}^{+n}+e^{-} \rightarrow \mathrm{A}^{+n-1}+h \nu$; dielectronic recombination, $\mathrm{A}^{+n}+e^{-} \rightarrow \mathrm{A}^{*++n-1} \rightarrow \mathrm{A}^{+n-1}+h \nu$; and charge-transfer recombination, $\mathrm{A}^{+n}+\mathrm{H}^{0} \rightarrow \mathrm{A}^{+n-1}+\mathrm{H}^{+}$. This paper focuses on radiative recombination for the four isoelectronic sequences in which this process should be predominant at nebular $\left(T \lesssim 10^{4}\right.$ $\mathrm{K})$ temperatures. (When we refer to the radiative recombination for an atom/ion, we mean the recombination that forms this species; e.g., "radiative recombination for $\mathrm{He} \mathrm{I}$ " means the recombination from $\mathrm{He}$ II to $\mathrm{He} \mathrm{I}$.)

During the last two decades, the power-law fits to the radiative recombination rates (Aldrovandi \& Pequignot 1973, hereafter AP; Shull \& Van Steenberg 1982; Arnaud \& Rothenflug 1985; Landini \& Monsignori Fossi 1990, 1991) have been extensively used for astrophysical applications. The AP calculations were made for $\mathrm{He}, \mathrm{C}, \mathrm{N}, \mathrm{O}, \mathrm{Ne}, \mathrm{Mg}, \mathrm{Si}$, and $\mathrm{S}$. The total radiative recombination coefficient was considered as a sum of recombination coefficients to the ground level and to all the excited levels. The ground-level recombination coefficients were evaluated by use of the Milne relation (see, e.g., Bates \& Dalgarno 1962) from the photoionization cross sections known in the early seventies; for many species, extrapolations of the cross sections along isoelectronic sequences were necessary because of lack of data. For all the excited levels, the hydrogenic approximation was assumed. The calculated radiative recombination coefficients were fitted by

$$
\alpha_{r}(T)=A\left(\frac{T}{10^{4} \mathrm{~K}}\right)^{-\eta},
$$

where $T$ is temperature and $A$ and $\eta$ are the fitting parameters. Shull \& Van Steenberg (1982) calculated and fitted by equation (1) the radiative recombination rates for all $\mathrm{Fe}$ ions. They also interpolated the rate coefficients for $\mathrm{Ar}, \mathrm{Ca}$, and $\mathrm{Ni}$. Interpolations of the rates for $\mathrm{Al}, \mathrm{Na}, \mathrm{F}, \mathrm{P}, \mathrm{Cl}, \mathrm{K}, \mathrm{Ti}, \mathrm{Cr}, \mathrm{Mn}$, and
Co were done by Landini \& Monsignori Fossi (1990, 1991). Arnaud \& Rothenflug (1985) improved the AP and Shull \& Van Steenberg (1982) rates for recombination toward He-like, Li-like, and Be-like ions, using the same power-law fitting formula (eq. [1]).

Gould (1978) introduced corrections to the hydrogenic formula for the radiative recombination coefficients to the excited levels of nonhydrogenic species. He provided more accurate total radiative recombination coefficients for the first four ionization states of $\mathrm{C}, \mathrm{N}, \mathrm{O}, \mathrm{Ne}, \mathrm{Mg}, \mathrm{Si}, \mathrm{S}$, and $\mathrm{Ar}$ at $T=10^{4} \mathrm{~K}$ and for the neutral species of these elements at $T=10^{2} \mathrm{~K}$. Pequignot, Petitjean, \& Boisson ( 1991 ) used updated photoionization cross sections from the ground and first excited $(n=$ 2 and $n=3$ ) states of all ions of $\mathrm{He}, \mathrm{C}, \mathrm{N}, \mathrm{O}$, and $\mathrm{Ne}$ for calculation of the radiative recombination coefficients. All levels $n>3$ were assumed to be hydrogenic. The results were fitted by the four-parameter formula

$$
\alpha_{r}(T)=10^{-13} z \frac{a t^{b}}{1+c t^{d}} \mathrm{~cm}^{3} \mathrm{~s}^{-1}
$$

where $z$ is the ionic charge ( $z=1$ for recombination toward neutral state), $t=10^{-4} T / z^{2}$, and $a, b, c, d$ are the fitting parameters. These fits are valid for the temperature range $40 z^{2} \mathrm{~K} \leq T \leq 4 \times 10^{4} z^{2} \mathrm{~K}$. Arnaud \& Raymond (1992) calculated iron radiative recombination rates of $\mathrm{Fe} X V$ through $\mathrm{Fe}$ XXVI using the photoionization cross sections from the ground and all the excited states with $n \leq 5$ presented by Clark, Cowan, \& Bobrowicz (1986). The rate coefficients were fitted by a modified form of equation (1):

$$
\alpha_{r}(T)=A\left(\frac{T}{10^{4} \mathrm{~K}}\right)^{-\alpha-\beta \log _{10}\left(T / 10^{4} \mathrm{~K}\right)},
$$

where $A, \alpha$, and $\beta$ are the fitting parameters.

This paper is part of a series concerned with the basic data needed to fully simulate spectral formation by all stages of ionization of the first 30 elements. Reliable rates are needed over the full temperature range $3 \mathrm{~K} \leq T \leq 10^{9} \mathrm{~K}$. During the last years, new, extensive calculations of the photoionization cross sections have been performed and published. Verner \& Yakovlev (1995) presented a complete set of analytic fits to the partial 
Hartree-Dirac-Slater (HDS) photoionization cross sections for the ground-state shells of all atoms and ions of elements from $\mathrm{H}$ to $\mathrm{Zn}$. These fits are very accurate far from the ionization thresholds of the outer shells and at higher energies above the subsequent inner-shell ionization edges. However, the HDS method may give significant errors near the thresholds of the outer shells, especially for neutral and low-ionized species. Use of the fits to the HDS photoionization cross sections for evaluation of the radiative recombination rates may lead to large errors in the low-temperature rates for neutral and low-ionized species. The recent Opacity Project (OP) calculations (Seaton et al. 1992) give more accurate low-energy photoionization cross sections obtained by the $\boldsymbol{R}$-matrix method (Burke et al. 1971 ) for all atoms and ions of elements with atomic numbers $Z \leq 14$ and $Z=16,18,20,26$. Unfortunately, the OP calculations do not include high-energy photoionization cross sections; these are required for high-temperature radiative recombination rates. Moreover, the OP database TOPbase (Cunto et al. 1993) contains only total photoionization cross sections, which are not appropriate for use with the Milne relation.

For the most ionic species, the total recombination rates are dominated by dielectronic recombination (Burgess 1964; Nussbaumer \& Storey 1984, 1986, 1987). The exceptions are Hlike ions, which are not subject to dielectronic recombination at all, and He-like, Li-like, and Na-like species, which do not have low-lying autoionization states and therefore are not subject to low-temperature dielectronic recombination (the process discussed in the papers by Nussbamer \& Storey). Thus, the rates of radiative recombination toward He-like, Li-like, and Na-like ions described here should be the total recombination rate at nebular temperatures and need only to be supplemented by high-temperature dielectronic recombination.

\section{CALCULATIONS AND FITS}

In this paper, we present the calculations and analytic fits to the rates of radiative recombination toward $\mathrm{H}$-like, He-like, $\mathrm{Li}$ like, and Na-like ions of all elements from $\mathrm{H}$ through $\mathrm{Zn}(\mathrm{Z}=$ 30 ). For calculation of the ground-level recombination rate coefficients for He-like, Li-like, and Na-like ions, we used the updated version of the analytic fits to the photoionization cross sections (Verner et al. 1996), which give the corrected threshold cross sections and near-threshold cross-sectional behavior based on the OP data and ensure accurate high-energy cross sections by use of the fitting formula with the correct nonrelativistic asymptote. At high temperatures, the direct recombination to the ground state gives the largest contribution to the total radiative recombination rate coefficient.

For calculation of the rate coefficients to the excited levels with $n \leq 5$ of the 5 and more times ionized species, we used Clark et al. (1986) fits to the partial photoionization cross sections. Note that the formula given by Clark et al. (1986) fits the cross sections up to $10 E_{\text {th }}$ only, where $E_{\text {th }}$ is the ionization threshold energy, and does not ensure correct asymptotic behavior: the cross sections decrease too slowly with increasing energy. It leads to artificially large recombination rate coefficients for Na-like species at $T \sim 10^{8} \mathrm{~K}$. To avoid it, we substituted Clark et al.'s high-energy tails by fits $\propto E^{-3}$ from $10 E_{\text {th }}$ to $100 E_{\mathrm{th}}$ and by nonrelativistic asymptote $\propto E^{-3.5-l}$, where $l$ is a subshell orbital quantum number, above $100 E_{\mathrm{th}}$. This substitution changes the high-temperature rates for He-like and Li-like ions by less than $1 \%$ but improves the rate coefficients for Na-like species at $T \gtrsim 10^{8} \mathrm{~K}$.

For calculation of the radiative recombination rates to the excited levels with $n \leq 10$ of the first four ionization states, where the Clark et al. (1986) fits are not valid, we used the Gould (1978) method of correction for incomplete shielding. The required values of energy levels with $n \leq 10$ for $\mathrm{He} \mathrm{I}, \mathrm{Li} \mathrm{I}$, Li II, Be II, Be III, B III, B IV, C IV, Na I, Mg II, Al III, and Si IV were retrieved from TOPbase version 0.7 (Cunto et al. 1993). We used the hydrogenic approximation for levels with $n>10$ (the species listed above) and for levels with $n>5$ ( 5 and more times ionized species).

To estimate the uncertainty of the Gould method, we have made more detailed calculations of the radiative recombination rates for $\mathrm{He} \mathrm{I}$. This was motivated by the importance of this process in measurements of the primordial helium abundance (Reeves 1992). We used the OP He I partial photoionization cross sections (Fernley, Taylor, \& Seaton 1987) of 40 low-lying excited levels with $n<10\left(l<3 ;{ }^{1} S,{ }^{1} P^{o},{ }^{1} D,{ }^{3} S\right.$, ${ }^{3} P^{o},{ }^{3} \mathrm{D}$ levels) to obtain recombination rates to these levels. All levels with $n \geq 10$ were assumed hydrogenic. Then we compared the obtained total radiative recombination rates with those calculated by the Gould method. At $T \leq 10^{2} \mathrm{~K}$, the discrepancies between these two methods are less than $1 \%$, since the main contribution to the total rate at these temperatures comes from the recombination to highly excited hydrogenic levels. At $T>10^{2} \mathrm{~K}$, the discrepancies begin to increase with increasing temperature and reach a maximum of $10 \%$ at $T=$ $10^{5} \mathrm{~K}$. At higher $T$, the discrepancies decrease again since the main contribution to the total rate at high temperature comes from the ground-state recombination. Thus, we can estimate that the accuracy of the Gould method is not worse than $10 \%$. The comparison of experimental and fitted photoionization cross sections of neutrals $(\mathrm{He}, \mathrm{Li}, \mathrm{Na})$ reveals that the accuracy of the fits to the ground-state photoionization cross sections is also better than $10 \%$. Therefore, the absolute accuracy of the calculated total radiative recombination rates presented here is not worse than $10 \%$ for neutrals and must be better for ions.

Careful integration of the Milne relation and use of the photoionization cross sections that are accurate as near the thresholds as at high energies ensure the high accuracy of the total recombination rate coefficients in a wide range of temperature. We calculated all the rates from $3 \mathrm{~K}$ to $10^{10} \mathrm{~K}$ using nonrelativistic photoionization cross sections. However, at temperatures above $10^{8} \mathrm{~K}$, relativistic corrections may be important for high- $Z$ ions. As we have mentioned, the largest contribution to the total recombination at $T>10^{8} \mathrm{~K}$ comes from the recombination to the ground level. Our fits for the ground-level photoionization cross sections of $\mathrm{H}$ and $\mathrm{He}$ agree within $2 \%$ with the experimental cross sections (Veigele 1973) at energies up to $1 \mathrm{MeV}$. Thus, the given recombination rate coefficients are accurate at temperatures up to $10^{9} \mathrm{~K}$ for low- $Z$ ions. For high- $Z$ ions, $T=10^{8} \mathrm{~K}$ is a more secure upper limit.

We fitted all the total rate coefficients obtained in the temperature range from $3 \mathrm{~K}$ to $10^{10} \mathrm{~K}$ using the formula

$$
\alpha_{r}(T)=a\left[\sqrt{T / T_{0}}\left(1+\sqrt{T / T_{0}}\right)^{1-b}\left(1+\sqrt{T / T_{1}}\right)^{1+b}\right]^{-1},
$$

where $a, b, T_{0}$, and $T_{1}$ are the fitting parameters. Equation (4) ensures correct asymptotic behavior of the rate coefficients 
(Ferland et al. 1992) for low and high temperatures: $\alpha_{r}(T) \propto$ $T^{-0.5}$ at $T \ll T_{0} \ll T_{1}$, and $\alpha_{r}(T) \propto T^{-1.5}$ at $T \gg T_{1} \gg T_{0}$. The average accuracy of the fitting is better than $2 \%$ (maximum error is less than 3\%) for He-like ions and better than $4 \%$ (maximum error is less than 6\%) for all Li-like and Na-like species. The errors of fitting are smaller for highly ionized species. Note that although all the fits are accurate in comparison with the calculated data up to $10^{10} \mathrm{~K}$, the upper limits of validity $\left(10^{8} \mathrm{~K}\right.$ for high- $Z$ ions and $10^{9} \mathrm{~K}$ for low- $Z$ ions ) are determined by the nonrelativistic character of the calculations.

Because of its importance for cosmological problems, we present two fits for the $\mathrm{He}$ I rates that are calculated by the more detailed method (see above). The first fit is valid at $T \leq$ $10^{6} \mathrm{~K}$ (the rms average error is $2.5 \%$, maximum error $5 \%$ ). Note that the $\mathrm{He}$ I high-temperature dielectronic recombination rate at $T=10^{6} \mathrm{~K}$ is 2 orders of magnitude larger than the radiative recombination rate at this temperature. The second fit is valid for all temperatures from $T=3 \mathrm{~K}$ up to $T=10^{10} \mathrm{~K}$ (the rms average error is $5 \%$, maximum error $10 \%$ ). The errors of fitting for all species under study are not higher than the estimated absolute errors. Therefore, our fitting does not lead to any loss of accuracy in comparison with the calculated data.

We also fitted the recombination rate coefficients for all the $\mathrm{H}$-like ions. Generally, the rate coefficients for all hydrogenic species can be obtained from the $\mathrm{H}$ I rate coefficient by use of the relation

$$
\alpha_{r}(Z, T)=Z \alpha_{r}\left(1, T / Z^{2}\right) .
$$

However, for the user's convenience we provide the separate fits to all the hydrogenic species, which ensure the uniform accuracy of the fitting for all ions in the same temperature range $3-10^{10} \mathrm{~K}$. The rms relative errors of the fits for all hydrogenic species are less than $0.5 \%$; the maximum errors are less than $1 \%$.

The fit parameters are listed in Table 1 . We have made extensive comparisons of our fits with the fits provided by AP, Shull \& Van Steenberg (1982), Arnaud \& Rothenflug ( 1985), Landini \& Monsignori Fossi (1990, 1991), Pequignot et al. (1991), and Arnaud \& Raymond (1992). Our fits are in very

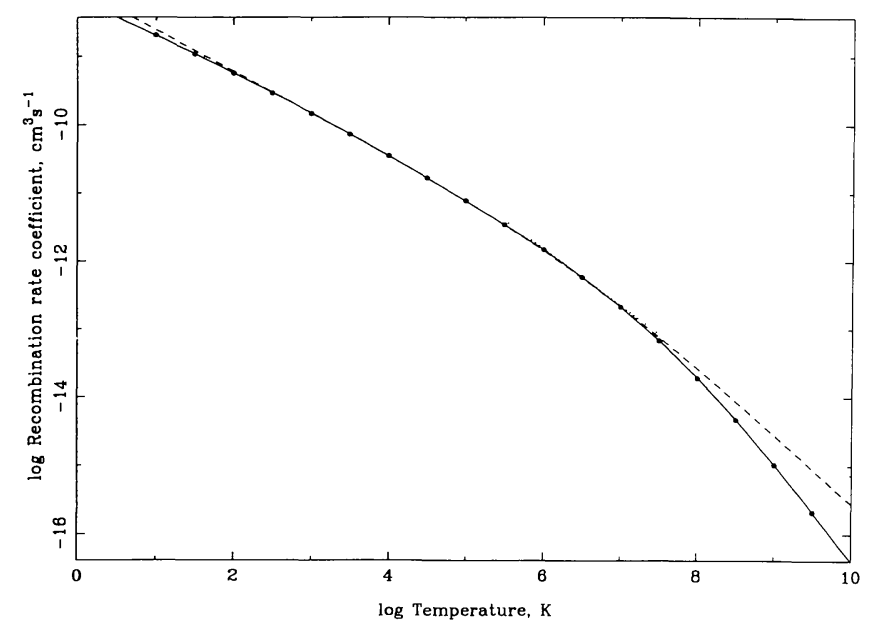

FIG. 1.-Radiative recombination rate coefficient of $\mathrm{O}$ vII vs. temperature. Circles: present calculations; solid line: present fit; dotted line: $\mathrm{Ar}-$ naud \& Rothenflug (1985); dashed line: Pequignot et al. (1991).

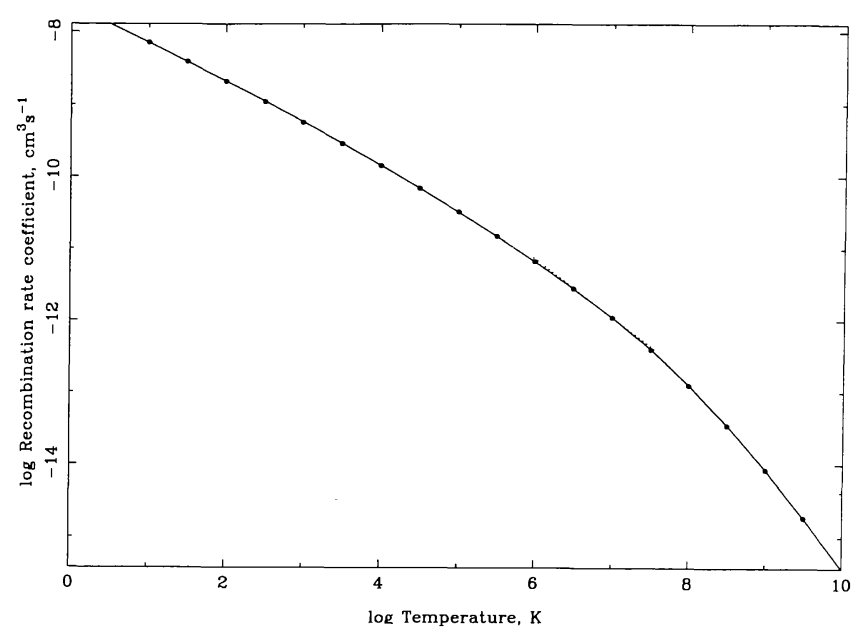

FIG. 2.-Same as Fig. 1, but for Si XIII

good agreement with the fits by Pequignot et al. (1991) and Arnaud \& Raymond (1992) in the temperature range in which the previous fits are valid. Our fits are accurate in a much wider temperature range, however, since our fitting formula has correct low-temperature and high-temperature asymptotes. The agreement with AP, Shull \& Van Steenberg (1982), Arnaud \& Rothenflug (1985), and Landini \& Monsignori Fossi (1990, 1991 ) is worse, especially for the cases interpolated along isoelectronic sequences in earlier papers. Figures 1-6 illustrate the comparison for the helium-like ions O VII and Si XIII, lithiumlike ions $\mathrm{C}$ IV and Fe XXIV, and sodium-like ions $\mathrm{Mg}$ II and Ca X. In Table 2, we compare the rates given by our fits with the rates provided by Gould (1978) at $T=10^{4} \mathrm{~K}$ for the three species that we have in common. The rates for these species obtained from the previously available fits are also shown.

For the hydrogenic species, Arnaud \& Rothenflug (1985) recommended the formula given by Seaton (1959):

$$
\begin{aligned}
\alpha_{r}(Z, T)=5.197 \times & 10^{-14} Z \lambda^{1 / 2} \\
& \times\left(0.4288+0.5 \ln \lambda+0.469 \lambda^{-1 / 3}\right),
\end{aligned}
$$

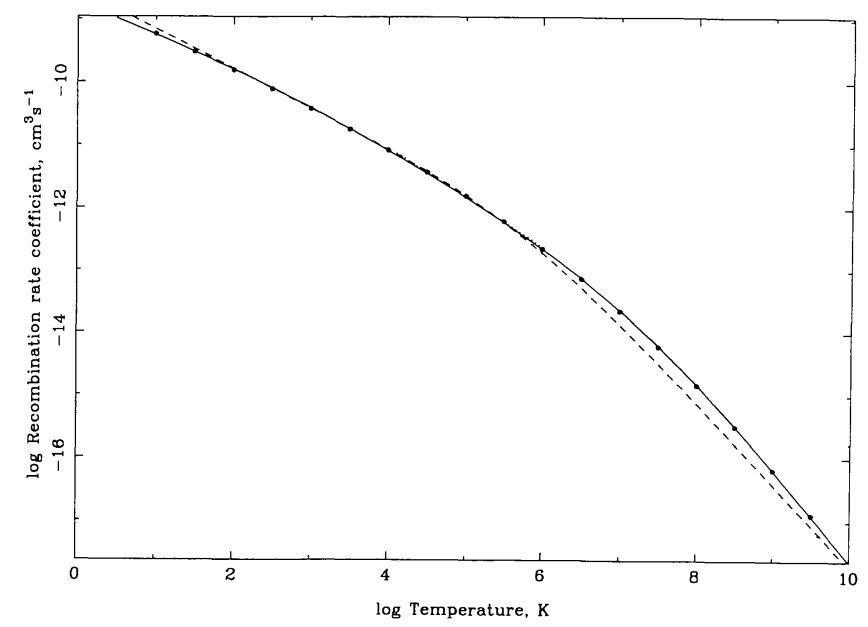

FIG. 3.-Same as Fig. 1, but for C IV 
TABLE 1

Fit PARAMETERS FOR RADIATIVE RECOMBINATION RATES

\begin{tabular}{|c|c|c|c|c|c|c|}
\hline Ion & $Z$ & $N$ & $a, \mathrm{~cm}^{3} \mathrm{~s}^{-1}$ & $b$ & $T_{0}, \mathrm{~K}$ & $T_{1}, \mathrm{~K}$ \\
\hline $\mathrm{H} \mathrm{I}$ & 1 & 1 & $7.982 \mathrm{e}-11$ & 0.7480 & $3.148 \mathrm{e}+00$ & $7.036 \mathrm{e}+05$ \\
\hline $\mathrm{He} \mathrm{I}^{\mathrm{a}}$ & 2 & 2 & $3.294 \mathrm{e}-11$ & 0.6910 & $1.554 \mathrm{e}+01$ & $3.676 \mathrm{e}+07$ \\
\hline $\mathrm{He} \mathrm{I}^{b}$ & 2 & 2 & $9.356 \mathrm{e}-10$ & 0.7892 & $4.266 \mathrm{e}-02$ & $4.677 \mathrm{e}+06$ \\
\hline He II & 2 & 1 & $1.891 \mathrm{e}-10$ & 0.7524 & $9.370 \mathrm{e}+00$ & $2.774 \mathrm{e}+06$ \\
\hline $\mathrm{Li} \mathrm{I}$ & 3 & 3 & $1.036 \mathrm{e}-11$ & 0.3880 & $1.077 \mathrm{e}+02$ & $1.177 \mathrm{e}+07$ \\
\hline Li II & 3 & 2 & $1.112 \mathrm{e}-10$ & 0.6926 & $2.437 \mathrm{e}+01$ & $8.323 e+06$ \\
\hline & 3 & 1 & $3.039 \mathrm{e}-10$ & 0.7539 & $1.871 \mathrm{e}+01$ & $6.209 \mathrm{e}+06$ \\
\hline Be II & 4 & 3 & $2.338 \mathrm{e}-11$ & 0.4211 & $3.647 \mathrm{e}+02$ & $1.215 \mathrm{e}+07$ \\
\hline Be III & 4 & 2 & $1.317 \mathrm{e}-10$ & 0.6691 & $8.473 e+01$ & $1.412 \mathrm{e}+07$ \\
\hline Be IV & 4 & 1 & $4.290 \mathrm{e}-10$ & 0.7557 & $3.000 \mathrm{e}+01$ & $1.093 \mathrm{e}+07$ \\
\hline B III & 5 & 3 & $4.487 \mathrm{e}-11$ & 0.4644 & $5.371 \mathrm{e}+02$ & $1.465 \mathrm{e}+07$ \\
\hline B IV & 5 & 2 & $1.922 \mathrm{e}-10$ & 0.6717 & $1.272 \mathrm{e}+02$ & $1.975 \mathrm{e}+07$ \\
\hline B V & 5 & 1 & $5.437 \mathrm{e}-10$ & 0.7560 & $4.576 \mathrm{e}+01$ & $1.706 \mathrm{e}+07$ \\
\hline C IV & 6 & 3 & $8.540 \mathrm{e}-11$ & 0.5247 & $5.014 \mathrm{e}+02$ & $1.479 e+07$ \\
\hline C V & 6 & 2 & $2.765 e-10$ & 0.6858 & $1.535 \mathrm{e}+02$ & $2.556 \mathrm{e}+07$ \\
\hline C VI & 6 & 1 & $6.556 \mathrm{e}-10$ & 0.7567 & $6.523 e+01$ & $2.446 \mathrm{e}+07$ \\
\hline $\mathrm{N} \mathrm{V}$ & 7 & 3 & $1.169 \mathrm{e}-10$ & 0.5470 & $6.793 e+02$ & $1.650 \mathrm{e}+07$ \\
\hline N VI & 7 & 2 & $3.910 \mathrm{e}-10$ & 0.6988 & $1.611 \mathrm{e}+02$ & $3.271 \mathrm{e}+07$ \\
\hline N VII & 7 & 1 & $7.586 \mathrm{e}-10$ & 0.7563 & $9.015 e+01$ & $3.338 \mathrm{e}+07$ \\
\hline O VI & 8 & 3 & $2.053 e-10$ & 0.6019 & $4.772 \mathrm{e}+02$ & $1.711 \mathrm{e}+07$ \\
\hline O VII & 8 & 2 & $4.897 \mathrm{e}-10$ & 0.7048 & $1.906 \mathrm{e}+02$ & $4.093 e+07$ \\
\hline O VIII & 8 & 1 & $8.616 \mathrm{e}-10$ & 0.7563 & $1.191 \mathrm{e}+02$ & $4.352 \mathrm{e}+07$ \\
\hline F VII & 9 & 3 & $2.739 e-10$ & 0.6188 & $5.033 e+02$ & $2.064 \mathrm{e}+07$ \\
\hline F VIII & 9 & 2 & $5.602 \mathrm{e}-10$ & 0.7052 & $2.476 \mathrm{e}+02$ & $5.077 \mathrm{e}+07$ \\
\hline F IX & 9 & 1 & $9.712 \mathrm{e}-10$ & 0.7566 & $1.499 \mathrm{e}+02$ & $5.498 \mathrm{e}+07$ \\
\hline $\mathrm{Ne}$ VIII & 10 & 3 & $3.200 \mathrm{e}-10$ & 0.6198 & $6.329 \mathrm{e}+02$ & $2.616 \mathrm{e}+07$ \\
\hline Ne IX & 10 & 2 & $6.161 \mathrm{e}-10$ & 0.7029 & $3.274 \mathrm{e}+02$ & $6.243 e+07$ \\
\hline $\mathrm{NeX}$ & 10 & 1 & $1.085 \mathrm{e}-09$ & 0.7570 & $1.834 \mathrm{e}+02$ & $6.776 e+07$ \\
\hline $\mathrm{Na} \mathrm{I}$ & 11 & 11 & $5.641 \mathrm{e}-12$ & 0.1749 & $3.077 \mathrm{e}+02$ & $2.617 \mathrm{e}+06$ \\
\hline $\mathrm{NaIX}$ & 11 & 3 & $3.873 \mathrm{e}-10$ & 0.6295 & $7.000 \mathrm{e}+02$ & $2.989 \mathrm{e}+07$ \\
\hline $\mathrm{NaX}$ & 11 & 2 & $6.833 e-10$ & 0.7018 & $4.060 e+02$ & $7.491 \mathrm{e}+07$ \\
\hline $\mathrm{Na} \mathrm{XI}$ & 11 & 1 & $1.163 \mathrm{e}-09$ & 0.7558 & $2.328 \mathrm{e}+02$ & $8.262 \mathrm{e}+07$ \\
\hline $\mathrm{Mg}$ II & 12 & 11 & $1.920 \mathrm{e}-11$ & 0.3028 & $4.849 \mathrm{e}+02$ & $5.890 \mathrm{e}+06$ \\
\hline $\operatorname{Mg} X$ & 12 & 3 & $4.284 \mathrm{e}-10$ & 0.6287 & $8.748 \mathrm{e}+02$ & $3.586 \mathrm{e}+07$ \\
\hline Mg XI & 12 & 2 & $7.510 \mathrm{e}-10$ & 0.7020 & $4.921 \mathrm{e}+02$ & $8.643 e+07$ \\
\hline Mg XII & 12 & 1 & $1.317 \mathrm{e}-09$ & 0.7574 & $2.585 \mathrm{e}+02$ & $9.769 e+07$ \\
\hline A1 III & 13 & 11 & $3.753 e-11$ & 0.3585 & $6.848 \mathrm{e}+02$ & $9.035 \mathrm{e}+06$ \\
\hline $\mathrm{Al} \mathrm{XI}$ & 13 & 3 & $4.881 \mathrm{e}-10$ & 0.6326 & $9.941 \mathrm{e}+02$ & $4.085 e+07$ \\
\hline Al XII & 13 & 2 & $8.182 \mathrm{e}-10$ & 0.7008 & $5.875 e+02$ & $1.007 \mathrm{e}+08$ \\
\hline Al XIII & 13 & 1 & $1.419 \mathrm{e}-09$ & 0.7578 & $3.057 \mathrm{e}+02$ & $1.143 \mathrm{e}+08$ \\
\hline Si IV & 14 & 11 & $5.942 \mathrm{e}-11$ & 0.3930 & $8.962 e+02$ & $1.213 e+07$ \\
\hline Si XII & 14 & 3 & $5.373 e-10$ & 0.6337 & $1.164 \mathrm{e}+03$ & $4.677 \mathrm{e}+07$ \\
\hline Si XIII & 14 & 2 & $8.722 e-10$ & 0.6996 & $7.098 \mathrm{e}+02$ & $1.155 \mathrm{e}+08$ \\
\hline Si XIV & 14 & 1 & $1.517 \mathrm{e}-09$ & 0.7574 & $3.601 \mathrm{e}+02$ & $1.329 \mathrm{e}+08$ \\
\hline P V & 15 & 11 & $1.721 \mathrm{e}-10$ & 0.5429 & $2.848 \mathrm{e}+02$ & $3.975 \mathrm{e}+07$ \\
\hline P XIII & 15 & 3 & $5.876 \mathrm{e}-10$ & 0.6354 & $1.341 \mathrm{e}+03$ & $5.292 \mathrm{e}+07$ \\
\hline P XIV & 15 & 2 & $9.142 \mathrm{e}-10$ & 0.6961 & $8.682 \mathrm{e}+02$ & $1.335 \mathrm{e}+08$ \\
\hline P XV & 15 & 1 & $1.586 \mathrm{e}-09$ & 0.7560 & $4.327 \mathrm{e}+02$ & $1.534 \mathrm{e}+08$ \\
\hline S VI & 16 & 11 & $3.502 \mathrm{e}-10$ & 0.6266 & $1.532 \mathrm{e}+02$ & $1.755 \mathrm{e}+07$ \\
\hline S XIV & 16 & 3 & $6.571 \mathrm{e}-10$ & 0.6400 & $1.452 \mathrm{e}+03$ & $5.796 \mathrm{e}+07$ \\
\hline S XV & 16 & 2 & $9.692 \mathrm{e}-10$ & 0.6945 & $1.017 \mathrm{e}+03$ & $1.517 \mathrm{e}+08$ \\
\hline S XVI & 16 & 1 & $1.729 e-09$ & 0.7568 & $4.725 e+02$ & $1.746 \mathrm{e}+08$ \\
\hline $\mathrm{Cl}$ VII & 17 & 11 & $2.502 \mathrm{e}-10$ & 0.5580 & $5.303 e+02$ & $4.558 \mathrm{e}+07$ \\
\hline $\mathrm{Cl} \mathrm{XV}$ & 17 & 3 & $7.076 \mathrm{e}-10$ & 0.6397 & $1.653 \mathrm{e}+03$ & $6.555 \mathrm{e}+07$ \\
\hline Cl XVI & 17 & 2 & $1.021 \mathrm{e}-09$ & 0.6932 & $1.184 \mathrm{e}+03$ & $1.695 \mathrm{e}+08$ \\
\hline Cl XVII & 17 & 1 & $1.791 \mathrm{e}-09$ & 0.7565 & $5.591 \mathrm{e}+02$ & $1.972 \mathrm{e}+08$ \\
\hline Ar VIII & 18 & 11 & $2.862 \mathrm{e}-10$ & 0.5621 & $7.002 \mathrm{e}+02$ & $4.885 \mathrm{e}+07$ \\
\hline Ar XVI & 18 & 3 & $7.538 \mathrm{e}-10$ & 0.6388 & $1.889 \mathrm{e}+03$ & $7.306 e+07$ \\
\hline Ar XVII & 18 & 2 & $1.087 \mathrm{e}-09$ & 0.6936 & $1.329 \mathrm{e}+03$ & $1.880 \mathrm{e}+08$ \\
\hline Ar XVIII & 18 & 1 & $1.913 \mathrm{e}-09$ & 0.7567 & $6.175 \mathrm{e}+02$ & $2.212 \mathrm{e}+08$ \\
\hline
\end{tabular}


TABLE 1-Continued

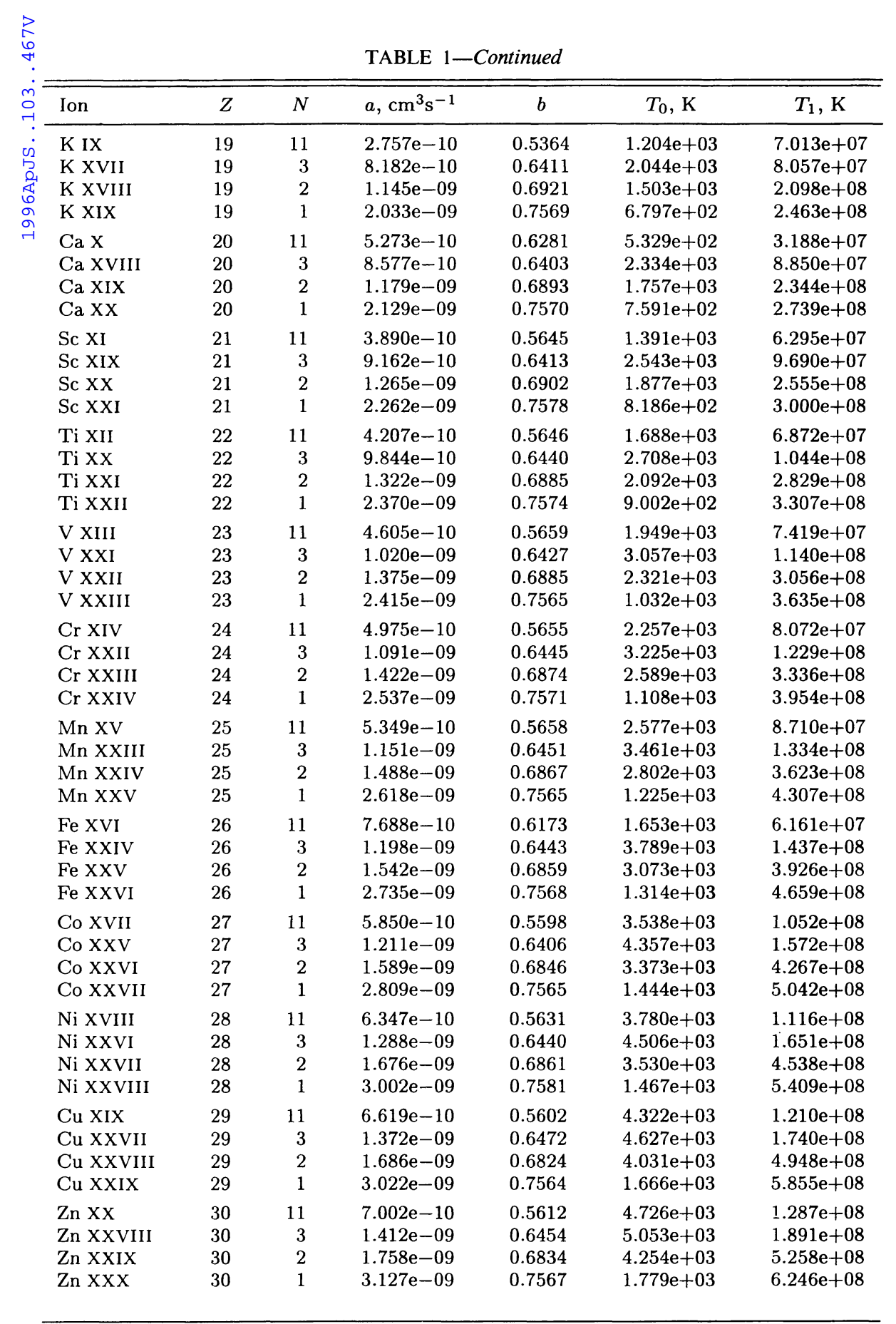

a Fit is valid at $3 \mathrm{~K} \leq T \leq 10^{6} \mathrm{~K}$, rms error $2.5 \%$.

${ }^{b}$ Fit is valid at $3 \mathrm{~K} \leq T \leq 10^{10} \mathrm{~K}$, rms error $4.7 \%$. 


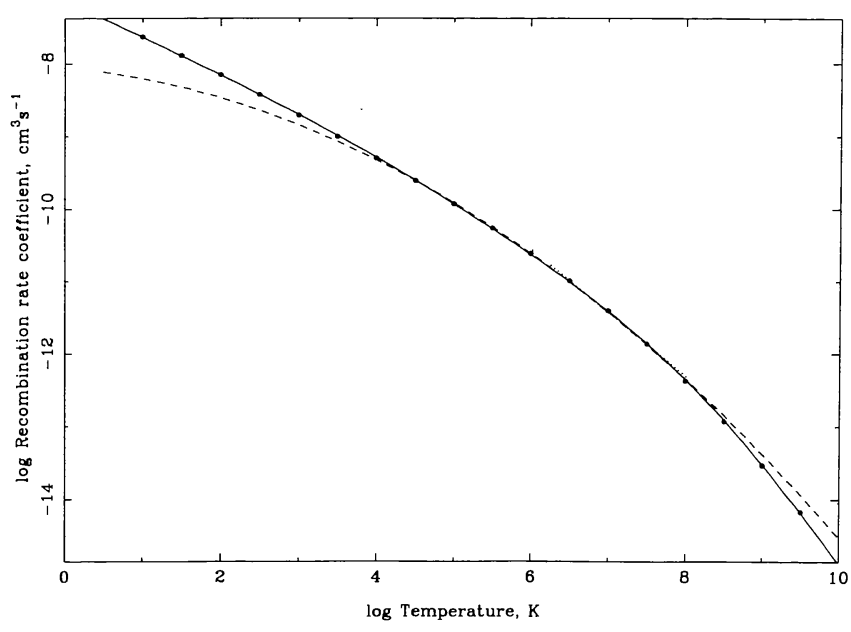

FIG. 4.- Same as Fig. 1, but for Fe XXIV. Dashed line: Arnaud \& Raymond (1992).

where $\lambda=157,890 Z^{2} / T(\mathrm{~K})$. However, this formula is not valid at high $\left(T>10^{6} Z^{2} \mathrm{~K}\right)$ temperatures. The hydrogenic fit given by Pequignot et al. (1991) also has an incorrect hightemperature asymptote. Figures 7 and 8 compare our hydrogenic data and fits with the previously available fits.

It can be useful for astrophysical applications to separate the partial recombination rate to the ground state and the recombination rate to all the excited states. We have fitted the rates of radiative recombination to the ground states and the rates of radiative recombination to all the excited states by the same fitting formula (eq. [4]). The corresponding fit parameters are available from the authors upon request. The accuracy of fitting of the partial rate coefficients is generally slightly lower than that of the total radiative recombination rate coefficients.

\section{DISCUSSION}

Our long-term goal is to predict the complete spectrum emitted by the first 30 elements. Fits to the needed photoionization

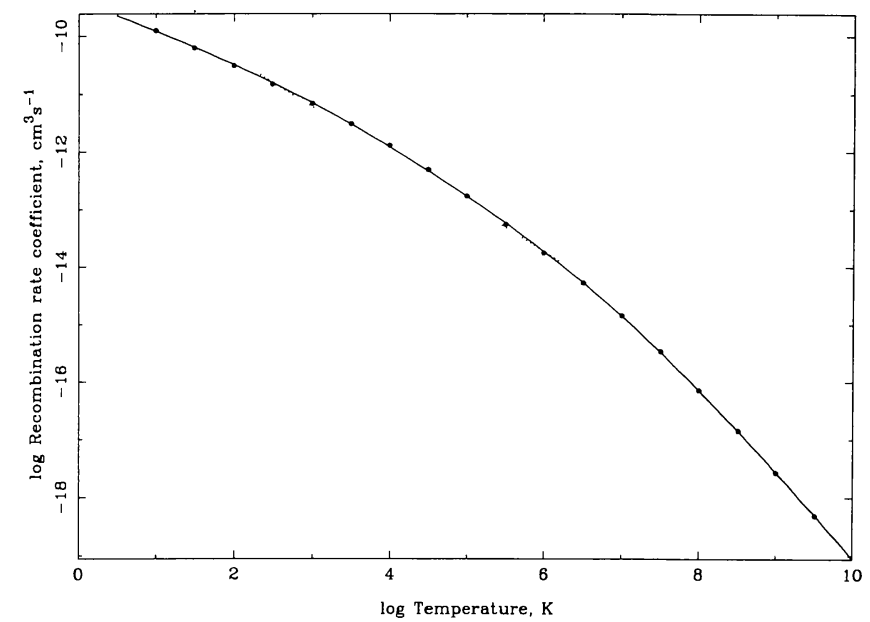

FIG. 5.-Same as Fig. 1, but for Mg II. Dotted line: Aldrovandi \& Pequignot (1973).

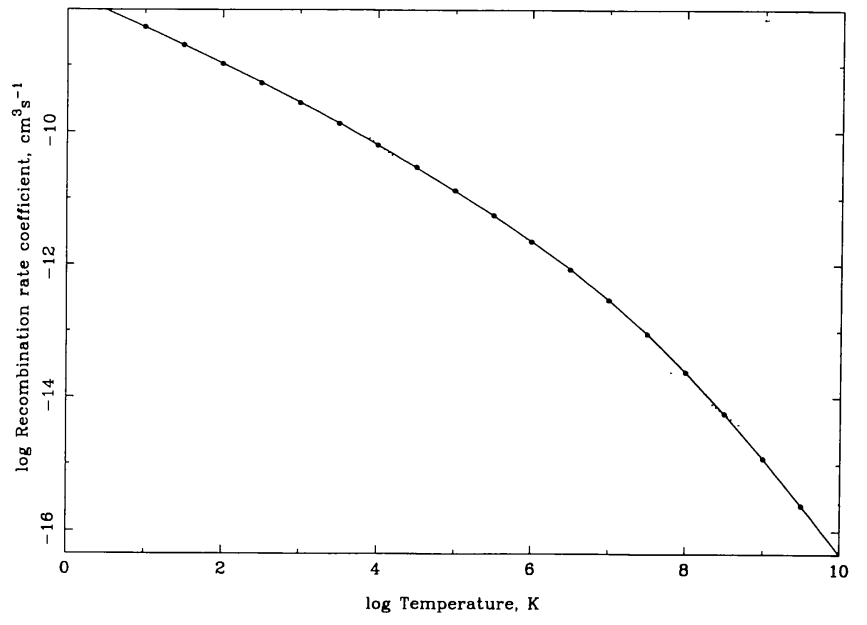

FIG. 6.-Same as Fig. 1, but for Ca X. Dotted line: Shull \& Van Steenberg (1982).

cross sections are presented by Verner \& Yakovlev (1995) and Verner et al. (1996). Here we present accurate fits to the total radiative recombination rates for 107 ions of four isoelectronic sequences, $23 \%$ of the rate coefficients needed for the 465 possible ions of the first 30 elements. This is the complete set of species for which simple radiative recombination is expected to be the dominant channel at nebular temperatures.

Our temperature range was selected with astrophysical needs in mind. We briefly mention two cases, at either temperature extreme, where the results presented here affect published results. In the low- $T$ extreme, charge transfer with $\mathrm{He}^{+}$is the dominant $\mathrm{CO}$ destruction mechanism in photodissociation regions (PDRs) for most conditions (Tielens \& Hollenbach 1985; Hollenbach \& McKee 1989; Ferland, Fabian, \& Johnstone 1994). The $\mathrm{He}^{+}$abundance is set by the balance between creation mechanisms (often ionization by cosmic rays or $\mathrm{X}$ rays) and radiative recombination. Our total rate coefficient for radiative recombination of $\mathrm{He}^{+}$to $\mathrm{He}$ at $300 \mathrm{~K}$, a representative PDR temperature, is $4.43 \times 10^{-12} \mathrm{~cm}^{3} \mathrm{~s}^{-1}$. This is $25 \%$ larger than the rate used by Hollenbach \& McKee (1989). Tests show that this does indeed result in a substantially greater $\mathrm{CO}$ abundance and cooling. At the opposite temperature extreme, heating by photoionization of hydrogenic species of the heavy elements is the main heating mechanism for a gas near $10^{6} \mathrm{~K}$. Our rates are often quite different from the power-law fits (AP; Shull \& Van Steenberg 1982; Arnaud \& Rothenflug

TABLE 2

RADIATIVE RECOMBINATION COEFFICIENTS AT $T=10^{4} \mathrm{~K}$

\begin{tabular}{cccc}
\hline \hline \multirow{2}{*}{ ION } & \multicolumn{3}{c}{ COEFFICIENT $\left(\mathrm{cm}^{3} \mathrm{~s}^{-1}\right)$} \\
\cline { 2 - 4 } & Gould 1978 & Present Fit & Old Fits $^{\mathrm{a}}$ \\
\hline C IV ........... & $8.45 \times 10^{-12}$ & $8.20 \times 10^{-12}$ & $9.16 \times 10^{-12}$ \\
Mg II ......... & $1.35 \times 10^{-12}$ & $1.22 \times 10^{-12}$ & $8.8 \times 10^{-13}$ \\
Si IV ........... & $7.32 \times 10^{-12}$ & $7.02 \times 10^{-12}$ & $5.5 \times 10^{-12}$ \\
\hline
\end{tabular}

a Arnaud \& Rothenflug 1985 for C IV, Aldrovandi \& Pequignot 1973 for $\mathrm{Mg}$ II and Si IV. 


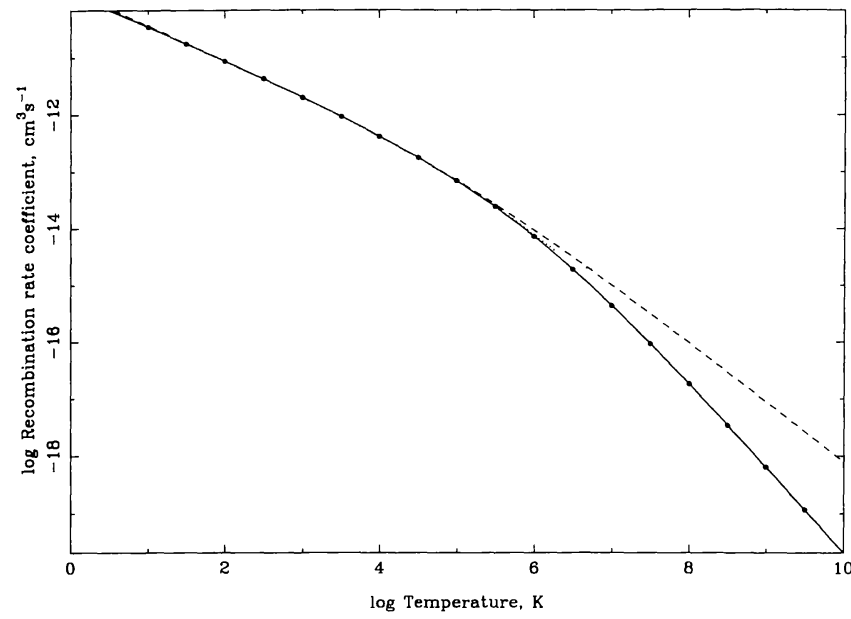

FIG. 7.-Radiative recombination rate coefficient of $\mathrm{H} I$ vs. temperature. Circles: present calculations; solid line: present fit; dotted line: Seaton (1959); dashed line: Pequignot et al. (1991).

$1985)$ extrapolated to high temperatures, and tests show that the photoelectric heating and resulting electron temperature tend to be lower, by as much as $20 \%$.

This work was supported by grants from the National Science Foundation (AST 9319034) and NASA (NAGW-3315). We are grateful to K. T. Korista, M. Arnaud, and D. G. Ya-

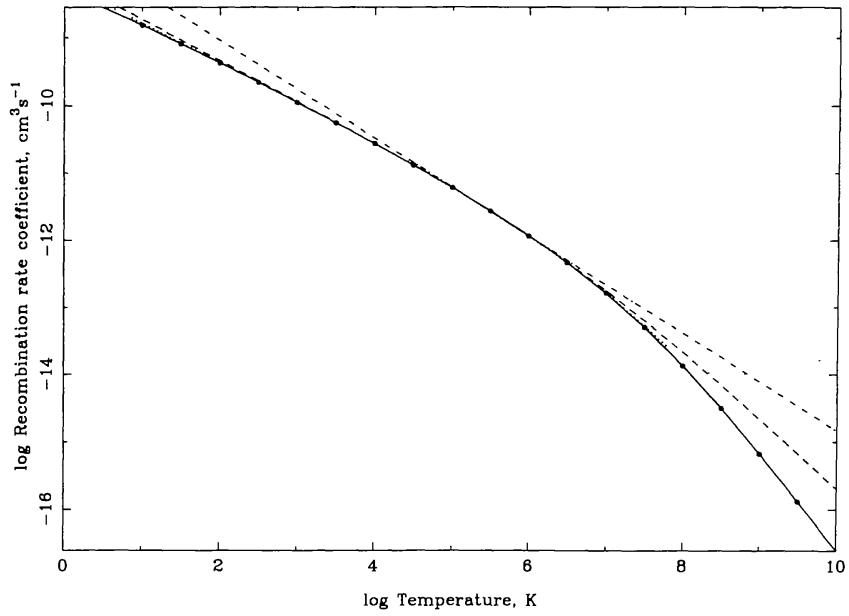

FIG. 8.-Same as Fig. 7, but for C VI. Dash-dotted line: Shull \& Van Steenberg (1982; see also erratum).

kovlev for useful discussions. We acknowledge the use of the TOPbase version 0.7 database installed by A. K. Pradhan at Ohio State University.

Table 1 and tables of the partial recombination rate coefficients in electronic form are available via anonymous ftp from asta.pa.uky.edu, directory $\operatorname{dima} / \mathrm{rec} /$, and through the World Wide Web page "Atomic Data for Astrophysics," 〈http: //www.pa.uky.edu/ verner/atom.html $\rangle$.

\section{REFERENCES}

Aldrovandi, S. M. V., \& Pequignot, D. 1973, A\&A, 25, 137; erratum 47, $321(1976)$ (AP)

Arnaud, M., \& Raymond, J. 1992, ApJ, 398, 394

Arnaud, M., \& Rothenflug, R. 1985, A\&AS, 60, 425

Bates, D. R., \& Dalgarno, A. 1962, in Atomic and Molecular Processes, ed.

D. R. Bates (New York: Academic), 245

Burgess, A. 1964, ApJ, 139, 776

Burke, P. G., Hibbert, A., \& Robb, W. D. 1971, J. Phys. B, 4, 153

Clark, R. E. H., Cowan, R. D., \& Bobrowicz, F. W. 1986, At. Data Nucl. Data Tables, 34, 415

Cunto, W., Mendoza, C., Ochsenbein, F., \& Zeippen, C. J. 1993, A\&A, 275, L5

Ferland, G. J., Fabian, A. C., \& Johnstone, R. M. 1994, MNRAS, 266, 399 Ferland, G. J., Peterson, B. M., Horne, K., Welsh, W. F., \& Nahar, S. N. 1992, ApJ, 387, 95

Fernley, J. A., Taylor, K. T., \& Seaton, M. J. 1987, J. Phys. B, 20, 6457

Gould, R. J. 1978, ApJ, 219, 250
Hollenbach, D. \& McKee, C. F. 1989, ApJ, 342, 306

Landini, M., \& Monsignori Fossi, B. C. 1990, A\&AS, 82, 229

- 1991, A\&AS, 91, 183

Nussbaumer, H., \& Storey, P. J. 1984, A\&AS, 56, 293 1986, A\&AS, 64, 545 1987, A\&AS, 69, 123

Pequignot, D., Petitjean, P., \& Boisson, C. 1991, A\&A, 251, 680

Reeves, H. 1992, in Elements and the Cosmos, ed. M. G. Edmunds \& R. J. Terlevich (Cambridge: Cambridge Univ. Press), 1

Seaton, M. J. 1959, MNRAS, 119, 81

Seaton, M. J., et al. 1992, Rev. Mexicana Astron. Astrofis., 23, 19

Shull, J. M., \& Van Steenberg, M. 1982, ApJS, 48, 95; erratum 49, 351

Tielens, A. G. G. M., \& Hollenbach, D. 1985, ApJ, 291, 722

Veigele, W. J. 1973, At. Data, 5, 51

Verner, D. A., Ferland, G. J., Korista, K. T., \& Yakovlev, D. G. 1996, ApJ, in press

Verner, D. A., \& Yakovlev, D. G. 1995, A\&AS, 109, 125 